\title{
Airborne Lidar Ve Dted2 Verilerinde Yükseklik (H) Karşılaştırması
}

\begin{abstract}
Muzaffer NAVRUZ ${ }^{1^{*}}$
${ }^{1}$ Maden Tetkik ve Arama Genel Müdürlüğü,Jeofizik Etütleri Daire Başkanlığı,Ankara

(muzaffer.navruz@mta.gov.tr) ORCID ID 0000-0002-6345-9260

Öz

Uzaktan algılamada Airborne (havadan) lidar yöntemi ile nokta bulutu üretimi yeni bir ivme kazanmıştır. Airborne (Havadan) Lidar ile üretilen sınıflandırılmış nokta bulutu verilerinden üretilen sayısal Arazi Modeli (SAM) çok disiplinli mühendislik uygulamalarında kullanılmaktadır. Zaman verisi ile $4 \mathrm{D}(\mathrm{X}, \mathrm{Y}, \mathrm{H}, \mathrm{T})$ veri toplama yöntemi olan LiDAR (Light Detection and Ranging) klasik yöntemlere göre hızlı bir yöntemdir. Erişilemeyen noktalarda veri elde edilmesi bir üstünlük olarak görülmektedir. Harita Genel Komutanlığı'nın Bergama test bölgesinde iki ayrı yükseklikten (1200m ve 2600m) Optech firmasının Pegasus HA-500 ve Riegl firmasının LMS-Q1560 LIDAR sistemlerinin özel yazılımları ile sınıflandırılmış nokta bulutları elde edilmiştir. Bu veri seti ve DTED2 veri setlerinden Global Mapper 17 yazılımı kullanılarak 5m grid aralıklı yükseklik $(H)$ verileri elde edilmiş ve iki veri setinin karşılaştırılması yapılmışıır. Veri seti yersel ölçüler ile arazi uygulamasında karşılaştırılmıştır. Bu veri setinin ülkemize kazandırılması ile Mühendislik projelerinde kullanım talebi artacak ve uzun süreli uygulama projelerinde zaman ve maliyet azaltıcı bir yöntem olarak önemli katkıları olacaktır.
\end{abstract}

Anahtar Kelimeler: Lidar, Optech, Riegl,Nokta Bulutu

\section{Airborne Lidar And Dted2 Data Height (H) comparison}

\begin{abstract}
In remote sensing, point cloud production has gained a new impetus with the Airborne lidar method. Airborne is used in Digital Terrain Model (SAM) multidisciplinary engineering applications produced from classified point cloud data generated by Lidar. LiDAR (Light Detection and Ranging), which is a 4D (X, Y, H, T) data collection method with time data, is a faster method than classical methods. It is seen as an advantage to obtain data at unreachable points. Point clouds classified by Optech's Pegasus HA-500 and Riegl's LMS-Q1560 LIDAR systems were obtained at two different altitudes $(1200 \mathrm{~m}$ and $2600 \mathrm{~m})$ in the Bergama test area of the Map General Command. Using the Global Mapper 17 software from this data set and DTED2 data sets, height (H) data with $5 \mathrm{~m}$ grid spacings were obtained and two data sets were compared. The dataset was compared with terrestrial measurements with terrestrial measurements. By bringing this dataset to my country, the usage demands in engineering projects will increase and it will be an important contribution as a time and cost reduction method in long time application projects.
\end{abstract}

Keywords: Lidar, Optech, Riegl,Point Cloud,

\section{GíRiş}

Sayısal Arazi Modeli (SAM),yeryüzünün belirli aralıklarla gridlenerek tanımlı bir datuma indirgenmesini içeren ve bünyesinde yükseklikleri barındıran sayısal bir gösterim şeklidir.Sayısal arazi modeli oluşturmak için arazi üzerinde uygun aralıklarla dağıtılmış 3 boyutlu kontrol noktaları gerekmektedir.Kontol noktalarından faydalanılarak enterpolasyon yöntemlerinden

\footnotetext{
* Sorumlu Yazar

$\mathrm{Bu}$ çalışma yayınlanmamıştır.IX.Tufuab Teknik Sempozyumunda sözlü bildiri olarak sunulmuştur
} 
uygun olan bir yöntem tercihi ile yeni noktalar elde edilir.Elde edilen yeni noktalar ve kontrol noktaları ile birlikte topografya sayısal olarak görüntülenir.Çalışma alanına ait verilerin fazla olması,uygun enterpolasyon yönteminin tercihi ve belirlenen grid aralığ $\breve{1}_{\text {Sayısal arazi }}$ modeline etki eder.Yöntemin ekonomik olması,kısa sürede geniş alanlarda istenilen hassasiyette 4 boyutlu çok veri elde etmeye uygun olması Lidar yöntemi tercih nedenidir.

\section{HAVADAN LIDAR SISTEMI}

Lidar yönteminin (Şekil 1) sayısal arazi modeli üretimimde uygulanabileceğine yönelik çalışmalar $1990 \quad$ l1 $\quad$ y1llarda başlamıştır.(Petzold vd.1999). Lidar,Havadan (airborne),Yerden (terrestrial) ve Gezici (mobile) olarak 3 grupta sınıflandırılmaktadır.Havadan lidar da Şekil 1sistemle bütünleşik yapıda lazer tarayıc1,global konum belirleme sistemi (GNSS), cihazın veya aracın hızını dönme açısını ve dönüklük miktarlarını belirleyen ve ana işlemciye gönderen IMU (Inertial Measurement Unit) birimlerinden oluşmaktadır.

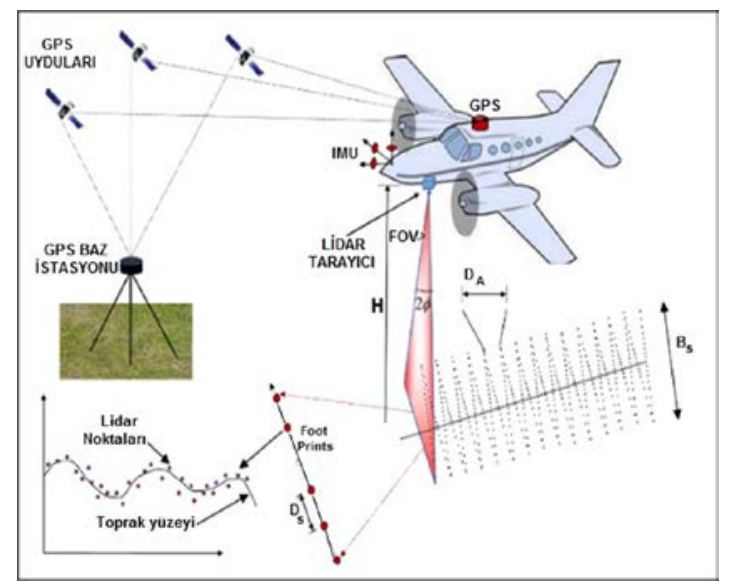

Şekil 1:Airborne Lidar

Bir hava taşıtına monte edilen sistem ile tarayıcının göderdiği 1şın demetinin zemine,bitki veya binalara çarptıktan sonra gidiş ve geliş sürelerini hesap eden algilayıc1lar yardımı ile mesafe hesaplanır. (Meng vd.,2010).Hesap edilen mesafe ile anlık konum tespit edilir ve kayıt altına alınır.
IMU ile de hava taşıtının konumu belirlenir.Hesaplanan mesafe ve hava taşıtının konum bilgisi yardımı ile zemindeki objelerin koordinat bilgileri elde edilir.Arazi yüzeyine saniyede oldukça fazla ışın demeti göndererek GPS ve IMU sayesinde zaman verisi ile birlikte 4 boyutlu veri üretilir.Lidar sistemi ile ,koordinat ve yükseklik ve zaman bilgilerini içeren çok fazla nokta üretilmektedir. Uygulamada topoğrafik haritalama,madencilik faaliyetleri,yer bilim araştırmaları,ormancılık,tarımsal

ugulamalar,yol projeleri,kent modelleme,kıy çizgisi değişiminin izlenmesi,demir yolu projelerinin uygulanması çok alanda yaygın olarak kullanılmaktadır.

\section{Araştırmanın Amacı}

Havadan Lidar verileri güncel teknoloji ile üretilen zaman boyutunu da içeren geometrik özellikli büyük boyutlu verilerdir.DTED2 verileri ise üretildiği zamanki teknoloji ile NATO STANAG MIL-PDF-89020B de belirtilen standartlarda mevcut 1/25.000 ölçekli topoğrafik haritalarda yer alan eş yüksek eğrileri, kot noktaları, göl ve deniz alanları kullanılarak üretilmiştir. Jeofizik yöntemlerin içerisinde Gravite yöntemi vardır. $\mathrm{Bu}$ yöntemin uygulamadaki en önemli sorunlarından biri Topografyadan gelen yükseklik hatalarıdır. Yersel ölçülerden gelen yükseklik hataları doğrudan ham gravite verileri ile Topoğrafik düzeltme (Terrain Correction) hesabında ilişkilendirildiğinden jeofizik ölçü sonucu elde edilen verilere olumsuz etki yapmaktadır. Ülkemizde yapılan Jeofizik araştırmaların sonucunda (KamuÖzel sektör) Topografik düzelmeler için kullanılan veri seti Harita Genel Komutanlığ (HGK) nın üretmiş olduğu Digital Terrain Elevation Data (DTED 2) vektör veri setidir.1 saniye (yaklaşık $30 \mathrm{~m}$ ) aralıklı üretilen bu verilerin $900 \mathrm{~m}^{2}$ lik $(30 \mathrm{~m} * 30 \mathrm{~m})$ alanında 4 adet nokta değeri varken aynı alanda 7200 Lidar nokta ( $\mathrm{m}^{2}$ de 8 nokta) verisi mevcuttur. Uygulamada test alanına ait DTED2 veri seti ile Lidar veri setleri karşılaştırılıp gereken yükseklik indirgemesi (Lidar veri kümesinin yükseklikleri elipsoidaldir.) yapıldıktan sonra ortaya çalışmaları etkileyecek düzeyde kot farklarının beklenmektedir.Yükseklik karsslastırılmasından yorumu etkileyecek düzeyde hata gelmesi öngörülmektedir. 


\section{YÖNTEM}

Karar verilen test alanı orman, yerleşim, su, tarımsal alan gibi detayların bulunduğu özellikleri dikkate alınarak puanlamaya tabi tutulmuştur. ( Harita Dergisi Ocak 2015 Sayı 153) (Tablo 1)Detay puanlama kriterleri.,(Tablo 2) Detaylara göre puanlanmasi.

Tablo 1: Detay Puanlama Kriterleri

\begin{tabular}{|c|l|}
\hline $\begin{array}{c}\text { Not } \\
\text { Değeri }\end{array}$ & \multicolumn{1}{c|}{ Açıklama } \\
\hline 3 & $\begin{array}{l}\text { "..." Test alanı ilgili ölçütü tam } \\
\text { olarak içermektedir. }\end{array}$ \\
\hline 2 & $\begin{array}{l}\text { ".." Test alanı ilgili ölçütü büyük } \\
\text { oranda içermektedir. }\end{array}$ \\
\hline 1 & $\begin{array}{l}\text { ".." Test alanı ilgili ölçütü az } \\
\text { miktarda içermektedir. }\end{array}$ \\
\hline 0 & $\begin{array}{l}\text { "..." Test alanı ilgili ölçütü } \\
\text { içermemektedir. }\end{array}$ \\
\hline
\end{tabular}

Tablo 2: Detaylara Göre Puanlanma.

\begin{tabular}{|c|c|c|c|c|c|c|c|}
\hline 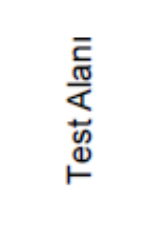 & 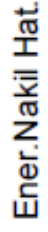 & ڤ్ & 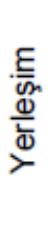 & ڤ & $\underset{\frac{E}{\sqrt{\sigma}}}{\stackrel{E}{\leftarrow}}$ & 希焉 & 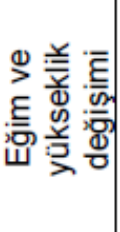 \\
\hline Bolu & 2 & 3 & 3 & 3 & 0 & 3 & 3 \\
\hline Aydın & 2 & 3 & 3 & 3 & 3 & 3 & 3 \\
\hline Bergama & 2 & 3 & 2 & 3 & 0 & 3 & 3 \\
\hline
\end{tabular}

Her bölge kurumlar tarafindan orman, yerleşim, su gibi içerdiği detaylara göre puanlanmıştır.( Harita Dergisi Ocak 2015 Say1 153)

Puanlama sonucu test alanının belirlenmesine karar verilmiş ve (Şekil 2) de belitilen Bergama test alanı Airborne Lidar için uygun bulunmuştur.

\section{Veri Toplama Araçları}

Araştırmada kullanılan veriler HARITA GENEL KOMUTANLIĞI (HGK) tarafindan Bergama test alanında toplanan Havadan Lidar verileri ile HGK tarafindan üretilen aynı bölgeye ait DTED2 veri setleri kullanılmıştır.

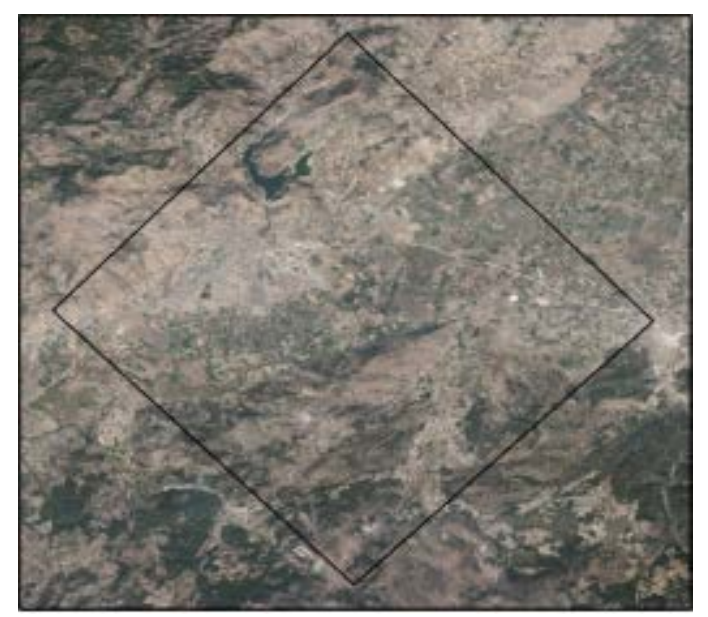

Şekil 2: Bergama Lidar Test Alanı

\section{Verilerin Analizi}

Bergama test alanında toplanan LIDAR verilerinden $1,9,49 \mathrm{~km}^{2}$ olmak üzere 3 farkl alan belirlenmiş ve $5 \mathrm{~m}$ gridli 3 vektör veri seti elde edilmiştir.Sınıflandırma parametreleri kulanılarak OPTECH Pegasus HA-500 ile 1200 m'den toplanan Lidar veri seti kullanılmıştır. Veri boyutunun büyüklüğü nedeniyle sadece $9 \mathrm{~km}^{2}$ lik alan verileri karşılaştırılmıştır.İ̧̧lem kolaylığı açısından saha test alanı verileri $1 * 1 \mathrm{~km}^{2}$ lik piksellere bölünmüştür. (Şekil 3) Parçalanmış Pikseller, (Şekil 4) Çalışma alanı veri sınırları

$$
\begin{aligned}
& \begin{array}{|c|c|c|c|}
\cline { 2 - 3 } \multicolumn{1}{c|}{} & 8 & 9 & \\
\hline 23 & 24 & 25 & 26 \\
\hline
\end{array}
\end{aligned}
$$

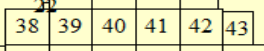

$$
\begin{aligned}
& \begin{array}{|lll|l|l|l|l|l|}
\hline 53 & 54 & 55 & 56 & 57 & 58 & 59 & 60 \\
\hline
\end{array} \\
& \begin{array}{|l|l|l|l|l|l|l|l|l|}
\hline 68 & 69 & 70 & 71 & 72 & 73 & 74 & 75 & 76 \\
\hline
\end{array} \\
& \begin{array}{r|r|l|l|l|l|l|l|l|l|}
\hline 384 & 85 & 86 & 87 & 88 & 89 & 90 & 91 & 92 & 93 \\
\hline 3
\end{array} \\
& \begin{array}{l|l|l|l|l|l|l|l|l|l|l|l|l|}
\hline 989 & 100 & 101 & 102 & 103 & 104 & 105 & 106 & 107 & 108 & 109 & 110 \\
\hline \text { th1 }
\end{array} \\
& \begin{array}{|l|l|l|l|l|l|l|l|l|l|l|l|l|l|}
\hline 114 & 115 & 116 & 117 & 118 & 119 & 120 & 121 & 122 & 123 & 124 & 125 & 126 & 127 \\
\hline
\end{array}
\end{aligned}
$$

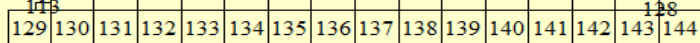

$$
\begin{aligned}
& \begin{array}{|l|l|l|l|l|l|l|l|l|l|l|l|l|l|l|l|}
\hline 145 & 146 & 147 & 148 & 149 & 150 & 151 & 152 & 153 & 154 & 155 & 156 & 157 & 158 & 159 & 160 \\
\hline
\end{array} \\
& \begin{array}{|r|l|l|l|l|l|l|l|l|l|l|l|l|l|l|l|l|l|l|}
\hline 161 & 162 & 163 & 164 & 165 & 166 & 167 & 168 & 169 & 170 & 171 & 172 & 173 & 174 & 175^{16}
\end{array}
\end{aligned}
$$

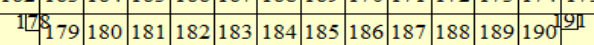

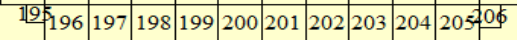

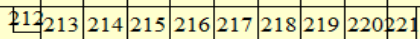

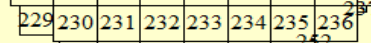

$$
\begin{aligned}
& 246247248249250251^{2}
\end{aligned}
$$

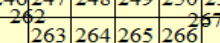

$$
\begin{aligned}
& 24928028282 \\
& 280281
\end{aligned}
$$

Şekil 3: Parçalanmış Pikseller

olmak üzere 3 alan seçilmiş ve bu alanlar için $5 * 5 \mathrm{~m}$ gridli veri setleri oluşturulmuştur. 


\begin{tabular}{|c|c|c|c|c|c|c|}
\hline 101 & 102 & 103 & 104 & 105 & 106 & 107 \\
\hline 117 & 118 & 119 & 120 & 121 & 122 & 123 \\
\hline 133 & 134 & 135 & 136 & 137 & 138 & 139 \\
\hline 149 & 150 & 151 & 152 & 153 & 154 & 155 \\
\hline 165 & 166 & 167 & 168 & 169 & 170 & 171 \\
\hline 181 & 182 & 183 & 184 & 185 & 186 & 187 \\
\hline 197 & 198 & 199 & 200 & 201 & 202 & 203 \\
\hline
\end{tabular}

Şekil 4: Lidar - DTED2 veri sınırları.

Uygulamada Test alanına ait DTED2 veri seti kullanılarak belirlenen alanlar için $5 \mathrm{~m}$ grid aralıklı vektör veri setleri üretilmiştir.Lidar veri kümesinin yükseklikleri elipsoidaldır.Çalışma alanına ait HGK tarafindan üretilen 1/25000 ölçekli pafta için Geoid $38.37 \mathrm{~m}$ dir. (Tablo-3) Yükseklik düzeltmesi.

Yükseklik verilerinin karşılaştırılmasından yorumu etkileyecek düzeyde yükseklik fark1 olması beklenmektedir.

Tablo 3: Yükseklik Düzeltmesi

\begin{tabular}{|c|c|c|c|c|c|}
\hline \multicolumn{5}{|c|}{ BALIKESIR J18-d3 Paftasına Ait Düzeltme } \\
Değerleri \\
\cline { 1 - 1 } \\
Pafta Adı & & & & & \\
\cline { 1 - 5 } BALIKESiR & & & & & \\
\hline J18-d3 & 3.69 & 1.73 & 186.3 & 41.6 & 38.37 \\
\hline
\end{tabular}

Airborne (Havadan) Lidar veri setinden elde edilen sınıflandırılmış nokta bulutu ile sadece zeminden gelen geri dönüş noktalarından hatalı noktalar ayıklanarak üretilen $5 \mathrm{~m}$ grid aralıklı Lidar vektör veri seti ile DTED2 verilerinin karşılaștırmaları ile elde edilen farklar (Tablo 4) de gösterilmiştir.
Tablo 4: LIDAR-DTED2 Vektör Veri Seti Farklar

\begin{tabular}{|lllllll|}
\hline Lidar & Lidar & & DTED2 & DTED2 & & Kot \\
& & & & & & \\
$(\mathrm{Y})$ & $(\mathrm{X})$ & Kot & $(\mathrm{Y})$ & $(\mathrm{X})$ & Kot & Fark1 \\
517970 & 4325325 & 162.18 & 517970 & 4325325 & 156.164 & 6.015 \\
517970 & 4325330 & 160.36 & 517970 & 4325330 & 154.233 & 6.126 \\
517970 & 4325335 & 159.03 & 517970 & 4325335 & 152.303 & 6.729 \\
517970 & 4325340 & 157.33 & 517970 & 4325340 & 151.576 & 5.755 \\
517970 & 4325345 & 155.87 & 517970 & 4325345 & 151.074 & 4.794 \\
517970 & 4325350 & 154.55 & 517970 & 4325350 & 150.572 & 3.974 \\
517970 & 4325355 & 153.08 & 517970 & 4325355 & 150.07 & 3.007 \\
517970 & 4325360 & 151.88 & 517970 & 4325360 & 149.568 & 2.316 \\
517970 & 4325365 & 150.66 & 517970 & 4325365 & 149.067 & 1.591 \\
517970 & 4325370 & 149.04 & 517970 & 4325370 & 147.837 & 1.207 \\
517970 & 4325375 & 147.49 & 517970 & 4325375 & 146.261 & 1.224 \\
517970 & 4325380 & 146.15 & 517970 & 4325380 & 144.685 & 1.461 \\
517970 & 4325385 & 144.73 & 517970 & 4325385 & 143.108 & 1.621 \\
517970 & 4325390 & 143.38 & 517970 & 4325390 & 141.53 & 1.845 \\
517970 & 4325395 & 142.1 & 517970 & 4325395 & 139.953 & 2.148 \\
\hline
\end{tabular}

Arazide Topcon GR5 GNSS alıcis1 kullanılarak TUSAGA-AKTiF ağ RTK yöntemiyle test alanında düz ve eğimli alanlarda olmak üzere iki farklı arazi tipinde ölçüler alınmıştır.Çalışma alanına ait $5 * 5 \mathrm{~m}$ grid aralıklı LIDAR veri seti aynı limit değerler kullanılarak karşılaştırılmıştır.Ölçü noktalarına en yakın komşu Lidar piksel değerleri alınarak Tablo 8 deki kot farkları elde edilmiştir.Elde edilen ölçü sonuçları ile LIDAR veri setleri arasındaki kot farklarının karşılaştırması sonucu Lidar verilerinin GNSS verileri ile $\sim 10 \mathrm{~cm}$ nin altında bir uyum sağladığı görülmektedir.(Tablo: 6,7,8,9,10,11)

Tablo 6 :Düz arazida yersel ölçüler

\begin{tabular}{|lccc|}
\hline NN & $\begin{array}{c}\text { GNSS } \\
(\mathrm{Y})\end{array}$ & $\begin{array}{c}\text { GNSS } \\
(\mathrm{X})\end{array}$ & $\begin{array}{c}\text { GNSS } \\
(\mathrm{H}) \mathrm{cm}\end{array}$ \\
BL1 & 518057.01 & 4328242.00 & 35.30 \\
BL2 & 518394.15 & 4328212.51 & 34.14 \\
BL3 & 518887.77 & 4328165.73 & 32.45 \\
BL4 & 519014.45 & 4327969.29 & 31.60 \\
BL5 & 519264.25 & 4328051.89 & 30.84 \\
BL6 & 519306.99 & 4327778.13 & 31.05 \\
BL7 & 519275.93 & 4327439.37 & 29.25
\end{tabular}


\begin{tabular}{|llll|} 
BL8 & 519093.21 & 4327092.42 & 30.62 \\
BL9 & 518896.40 & 4327416.29 & 31.10 \\
BL10 & 518588.01 & 4327485.64 & 31.57 \\
BL11 & 518346.58 & 4327542.11 & 32.08 \\
BL12 & 518050.25 & 4327701.78 & 32.37 \\
\hline
\end{tabular}

Tablo 7:Düz arazi 5*5 Gridli Lidar verileri

\begin{tabular}{|lccc|}
\hline NN & $\begin{array}{c}\text { LiDAR } \\
(\mathrm{Y})\end{array}$ & $\begin{array}{c}\text { LiDAR } \\
(\mathrm{X})\end{array}$ & $\begin{array}{c}\text { LíAR } \\
(\mathrm{H}) \mathrm{cm}\end{array}$ \\
BL1 & 518055.00 & 4328240.00 & 35.35 \\
BL2 & 518395.00 & 4328210.00 & 34.15 \\
BL3 & 518885.00 & 4328165.00 & 32.47 \\
BL4 & 519015.00 & 4327970.00 & 31.69 \\
BL5 & 519260.00 & 4328050.00 & 30.88 \\
BL6 & 519305.00 & 4327780.00 & 30.90 \\
BL7 & 519275.00 & 4327440.00 & 29.54 \\
BL8 & 519090.00 & 4327090.00 & 30.63 \\
BL9 & 518895.00 & 4327415.00 & 31.15 \\
BL10 & 518590.00 & 4327490.00 & 31.64 \\
BL11 & 518345.00 & 4327545.00 & 32.13 \\
BL12 & 518050.00 & 4327700.00 & 32.36 \\
\hline
\end{tabular}

Tablo 8: Düz arazide GNSS-Lidar kot farkları

\begin{tabular}{|llll|}
\hline NN & GNSS(H) & LIDAR(H) & $\begin{array}{c}\text { Kot } \\
\text { Fark1(cm) }\end{array}$ \\
BL1 & 35.30 & 35.35 & -0.05 \\
BL2 & 34.14 & 34.15 & -0.02 \\
BL3 & 32.45 & 32.47 & -0.02 \\
BL4 & 31.60 & 31.69 & -0.09 \\
BL5 & 30.84 & 30.88 & -0.04 \\
BL6 & 31.05 & 30.90 & 0.15 \\
BL7 & 29.25 & 29.54 & -0.29 \\
BL8 & 30.62 & 30.63 & -0.01 \\
BL9 & 31.10 & 31.15 & -0.06 \\
BL10 & 31.57 & 31.64 & -0.07 \\
BL11 & 32.08 & 32.13 & -0.05 \\
BL12 & 32.37 & 32.36 & 0.01 \\
\hline
\end{tabular}

Tablo 9: Eğimli arazi yersel ölçüler

\begin{tabular}{|lccc|}
\hline NN & $\begin{array}{c}\text { GNSS } \\
(\mathrm{Y})\end{array}$ & $\begin{array}{c}\text { GNSS } \\
(\mathrm{X})\end{array}$ & $\begin{array}{c}\text { GNSS } \\
(\mathrm{H}) \mathrm{cm}\end{array}$ \\
BL21 & 517966.47 & 4326194.79 & 27.968 \\
BL22 & 518306.70 & 4326198.51 & 28.421 \\
BL23 & 518653.30 & 4326235.30 & 28.859 \\
BL24 & 518836.80 & 4326234.91 & 29.067 \\
BL25 & 518755.98 & 4325956.99 & 61.283
\end{tabular}

\begin{tabular}{|llll|} 
BL26 & 518548.85 & 4325616.91 & 112.18 \\
BL27 & 518391.78 & 4325335.87 & 159.84 \\
BL28 & 518677.00 & 4325326.67 & 166.04 \\
BL29 & 518077.11 & 4325350.55 & 129.55 \\
BL30 & 518019.99 & 4325608.96 & 97.322 \\
BL31 & 518201.12 & 4325741.73 & 45.425 \\
BL32 & 518441.95 & 4325985.44 & 29.804 \\
BL33 & 518058.68 & 4325951.78 & 27.937 \\
\hline
\end{tabular}

Tablo 10: Eğimli arazide Lidar verileri

\begin{tabular}{|lccl|}
\hline NN & $\begin{array}{c}\text { Lidar } \\
(Y)\end{array}$ & $\begin{array}{c}\text { Lidar } \\
(\mathrm{X})\end{array}$ & \multicolumn{1}{c|}{$\begin{array}{c}\text { Lidar } \\
(\mathrm{H}) \mathrm{cm}\end{array}$} \\
BL21 & 517970.00 & 4326195.00 & 27.843 \\
BL22 & 518305.00 & 4326200.00 & 28.481 \\
BL23 & 518655.00 & 4326235.00 & 28.848 \\
BL24 & 518835.00 & 4326235.00 & 29.181 \\
BL25 & 518755.00 & 4325955.00 & 61.474 \\
BL26 & 518550.00 & 4325615.00 & 112.442 \\
BL27 & 518390.00 & 4325335.00 & 159.687 \\
BL28 & 518675.00 & 4325325.00 & 165.962 \\
BL29 & 518080.00 & 4325350.00 & 129.606 \\
BL30 & 518015.00 & 4325610.00 & 97.364 \\
BL31 & 518200.00 & 4325740.00 & 45.3 \\
BL32 & 518445.00 & 4325985.00 & 29.89 \\
BL33 & 518060.00 & 4325950.00 & 27.919 \\
\hline
\end{tabular}

Tablo 11:Eğimli arazi GNSS-Lidar kot fark1

\begin{tabular}{|lllc|}
\hline NN & GNSS & Lidar & $\begin{array}{c}\text { Kot } \\
\text { Farkı }(\mathrm{cm})\end{array}$ \\
BL21 & 27.968 & 27.843 & 0.13 \\
BL22 & 28.421 & 28.481 & -0.06 \\
BL23 & 28.859 & 28.848 & 0.01 \\
BL24 & 29.067 & 29.181 & -0.11 \\
BL25 & 61.283 & 61.474 & -0.19 \\
BL26 & 112.182 & 112.442 & -0.26 \\
BL27 & 159.838 & 159.687 & 0.15 \\
BL28 & 166.044 & 165.962 & 0.08 \\
BL29 & 129.554 & 129.606 & -0.05 \\
BL30 & 97.322 & 97.364 & -0.04 \\
BL31 & 45.425 & 45.3 & 0.13 \\
BL32 & 29.804 & 29.89 & -0.09 \\
BL33 & 27.937 & 27.919 & 0.02 \\
\hline
\end{tabular}




\section{BULGULAR}

Elde edilen veri setlerinin yüksekliklerinin karşılaştırılması sonucu Havadan lidar verileri ile DTED2 verilerinin aynı limit değerleri ve aynı grid aralıklarında çalışma alanında $6 \mathrm{~m}$ ye kadar varan yükseklik farklarının olduğu görülmektedir.

\section{SONUÇLAR}

Yersel ölçülerin Lidar verileri ile karşılaştırılması sonucu Lidar verilerinin GNSS verileri ile $\sim 10 \mathrm{~cm}$ nin altında bir uyum sağladığı görülmektedir.Topografyanın bozuk yükseltinin fazla olduğu bölgelerde DTED2LIDAR veri test alanının tamamı dikkate alındığında $\pm 12 \mathrm{~m}$ ye kadar varan kot farkları olurken seçilen 3 alanda kot farkları 6 m'yi bulmaktadır (Tablo 4).Bu farklar Jeofizik Mikrogravite yöntem ile yapılacak çalışmalara önemli katkılar sağlayacaktır. Mikrogravite yöntemi ile yapılan topografik tashih (Terrain Correction) hesaplamalarında ham jeofizik verileri ile ilişkilendirilen bu yükseklik farkının oluşturacağı 1-2 mgal büyüklüğündeki değer detay maden arama, zemin etütleri, obruk araştırmaları, arkeojeofizik etütlerde çok önem kazanmaktadır. Erişilmesi zor alanlarda yapılacak çalışmalar için önemli bir kolaylık sağlayan yöntemin kullanımı ile özellikle büyük ve geniş alanlarda, şeritvari harita üretimi, koridor, elektrik hatları iletimi ve her türlü haritacılık faaliyetlerinde kısa zamanda çok iş üretimine erişildiği için tercih edileceği düşünülmektedir. Test alanı yaklaşık $150 \mathrm{~km}^{2}$ dir. Bu alanın klasik yöntemlerle harita üretim amaçlı ölçümü uzun zaman alacaktır.

\section{TEŞEKKÜR}

Havadan Lidar verilerini Üniversitelerimiz ,kamu kurum ve kuruluşlarımız için Ülkemize kazandırılmasının test edilmesi amaciyla üretimini sağlayan HARITA GENEL KOMUTANLIĞIMIZA, NIK İnşaat Ticaret Ltd.Şti (Optech Pegasus HA-500 LIDAR sistemi) ve SEZA Teknik Cihazlar Ltd.Şti (Riegl LMS-Q1560 Lidar Sistemi) ne,Arazi çalışmasına destek veren M.T.A. GENEL MÜDÜRLÜĞÜNE, Karşılaştırmalı yükseklik verilerinin Jeofizik Gravite yönteminde kullanılabilirliğini yorumlayan Maden Tetkik ve Arama Genel Müdürlüğü, Jeofizik Etütleri Dairesi Başkanlığı elemanlarından Dr. Muzaffer Özgü Arısoy,Jeofizik Yük. Müh. Atakan ALACA'ya, Gravita-Manyetik Birim Yöneticisi Jeofizik Müh . Aytekin AYVA 'ya, Arazi çalışmalarında katkı koyan Harita Mühendisi Aydın Ayranc1,İzzettin Alan ve Hasan İlden'e teşekkür ederim.

NOT: $\mathrm{Bu}$ çalışma Afyon Kocatepe Üniversitesinde 27- 29 Nisan 2017 tarihleri arasında gerçekleştirilen TUFUAB IX. Teknik Sempozyumununda sunula ve yeniden hakemlik sürecinden geçirilerek Kabul edilmiş halidir.

\section{KAYNAKÇA}

Abdullah KAYI , Mustafa ERDOĞAN, Oktay EKER Harita Genel Komutanlığ 06240 Dikimevi, Ankara, abdullah.kayi@hgk.msb.gov.tr Harita Dergisi Ocak 2015 Say1 153

A.Yılmaz, O.Alp, A.OKUL, O.Eker, M.Erdoğan Harita Genel Komutanlığı, Tip Fakültesi Caddesi 06590 Cebeci/ANKARA

altan.yilmaz, osman.alp, abdullah.okul, oktay.eker,

mustafa.erdogan@hgk.msb.gov.tr TÜRKIYE ICÇIN HASSAS YÜKSEKLIK MODELİ ÜRETIMI

BHIKPK, (2014),Bakanlıklararası Harita İşlerini Koordinasyon ve Planlama Kurulunun Bilimsel Araştırma ve koordinasyon Komisyonu 2013-2014 Faaliyet Raporu

Meng X., Currit N., Zhao, K., (2010), Ground filtering algorithms for airborne LiDAR data: A review of critical issues, Remote Sensing, 2 (3), 833า860

Petzold B., Reiss P., Stossel W., (1999), Laser scanning $\neg$ surveying and mapping agencies are using a new technique for the derivation of digital terrain models, ISPRS Journal of Photogrammetry and Remote Sensing, 54, 95-104.

$\operatorname{Url}(1)$

http://www.mta.gov.tr/v3.0/sayfalar/hizme tler/kutuphane/ekonomibultenleri/2011_11/bulten11.pdf

$\operatorname{Url}(2)$

https://ai2-s2-

public.s3.amazonaws.com/figures/2016-108/52a5bc4aace403cd220fa86cb0334cbf4 08fb455/3-Figure1-1.png 